\title{
Anthropogene Umweltveränderungen in den Südtiroler Ötztaler Alpen in historischer Zeit
}

\section{Max Stumböck, Augsburg}

\section{Einleitung}

Mit Beginn des Holozäns um 9000 v. Chr. kann der mesolithische Mensch sein Siedlungsareal auch in den Zentralalpen bedeutend ausweiten (Lunz 1986). Seither hat der Mensch die alpine Umwelt für seine Zwecke umgestaltet, ein Prozeß, der in natürlichen Archiven - vor allem aus Seen und Mooren - konserviert ist. Später geben dann schriftliche Quellen zusätzliche Informationen.

Die vorliegende Studie untersucht das Wirken des Menschen in historischer Zeit anhand von Beispielen aus der montanen und subalpinen Höhenstufe der Südtiroler Ötztaler Alpen - ein Raum, der im Forschungsstand als «tabula rasa» zu bezeichnen ist.

\section{Naturraum}

Die Ötztaler Alpen auf Südtiroler Seite umfassen den nördlichen Vinschgau bis zum Alpenhauptkamm mit den großen Seitentälern Matscher Tal, Schnalstal und Passeiertal (Abb. 1). Nach Ozenda (1988) gehört der Raum zum inneralpinen Zentrum der Ostalpen westlich des Brenners. Dieser wird naturräumlich charakterisiert durch vorherrschende Hochlagen oberhalb 2000 m ü.M., durch die Ötztaler-Stubaier Masse mit silikatischem Gesteinsbestand und durch ein kontinentales Klima mit ausgeprägter thermischer Gunstlage (Massenerhebungseffekt). So betragen die langjährig gemittelten Niederschläge am Eingang des Schnalstals $495 \mathrm{~mm}$ (Station Naturns, $554 \mathrm{~m}$ ü.M.) und im mittleren Schnalstal nur $629 \mathrm{~mm}$ (Station Vernagt, $1700 \mathrm{~m}$ ü.M.) (Hydrographisches Amt 1994). Die Lufttemperaturen sind für Naturns $10.7^{\circ} \mathrm{C}$ und für Vernagt noch $5.9^{\circ} \mathrm{C}$ im langjährigen Mittel (HydroGRAPHISCHES Амт 1995). Die dominante Vegetation der montanen Höhenstufe ist Fichtenwald, der im subalpinen Bereich von Lärchen- und Zirbenbeständen abgelöst wird. Die Zirbe bildet dann die aktuelle Waldgrenze in Höhen von 2000 bis $2200 \mathrm{~m}$ ü.M., die Baumgrenze liegt etwa $150 \mathrm{~m}$ darüber. Als absolutes Höhenmaximum wurde im inneren Schnalstal in geschützter Südexposition eine $1.5 \mathrm{~m}$ hohe Zirbe auf $2520 \mathrm{~m}$ ü.M. gefunden. Oberhalb der subalpinen Wälder schließen sich die alpine und nivale Höhenstufe mit Zwergsträuchern, Rasen und Pioniervegetation an.

\section{Methodik}

Methodische Basis der vorliegenden Studie bilden Sedimente, die aus sechs Feuchtgebieten der hochmontanen, subalpinen und alpinen Höhenstufe der Südtiroler Ötztaler Alpen gewonnen wurden. Die Auswahl erfolgte nach umfangreichen Begehungen, bei denen alle im Gebiet bekannten 44 Standorte aufgesucht und tiefensondiert wurden (Zusammenstellung in Stumböck 1999). Wesentliche Hilfe war dabei der «Kataster der Moore und Feuchtgebiete Südtirols» (Biologisches LANDESLABOR 1991) sowie Orthofotokarten 1:10000, die flächendeckend vorliegen (Амт FÜR ÜBERÖRTLICHE RAUMORDNUNG).

Die 1.7 bis $5.7 \mathrm{~m}$ tiefen Bohrkerne der ausgewählten sechs Standorte wurden pollenanalytisch, dendrologisch und sedimentstratigraphisch detailliert bearbeitet. Dabei wurden die Sedimente mittels Standardmethoden aufbereitet und analysiert (z.B. MOoRE et al. 1991; SCHWEINGRUBer 1990; Merkt et al. 1971; SCHNEeKLOTH \& SCHNEIDER 1972). Ergänzt wurden die Untersuchungen durch AMS-Radiokarbondatierungen (R.J. Van de Graaff Laboratorium der Universität Utrecht) und durch archivalische Arbeiten bzw. Literaturstudien zur Siedlungs- und Nutzungsgeschichte im Tiroler Landesmuseum Ferdinandeum, Innsbruck.

\section{Natürliche Umweltveränderungen im Spätglazial und Holozän}

Die wichtigsten Parameter zur Klima- und Vegetationsgeschichte der Ötztaler Alpen sind in Abb. 2 verzeichnet und skizzieren die naturräumlichen Rahmenbedingungen für das Einwandern und das Wirken des Menschen im Alpenraum seit dem Rückschmelzen der würmzeitlichen Eisbedeckung. Die Ergebnisse wurden vor allem auf der Basis von glazialmorphologischen, glaziologischen und pollenanalytischen Befunden gewonnen.

Für den Ostalpenraum wird die maximale Eisbedekkung im Hauptwürm mit 20000 v.h. angegeben. Die darauffolgenden 5000 Jahre sind dann durch einen starken Eiszerfall geprägt (HANTKE 1983). Während der frühen Phase der Ältesten Dryas liegen die Temperaturen etwa um $9 \mathrm{~K}$ unter den heutigen. Dieser Wert wird nach PATzelt (1975), bestätigt von BoRTENSCHLAGER (1991), aus den anhand von Moränen rekonstruierten Gletscherständen im Ostalpenraum 


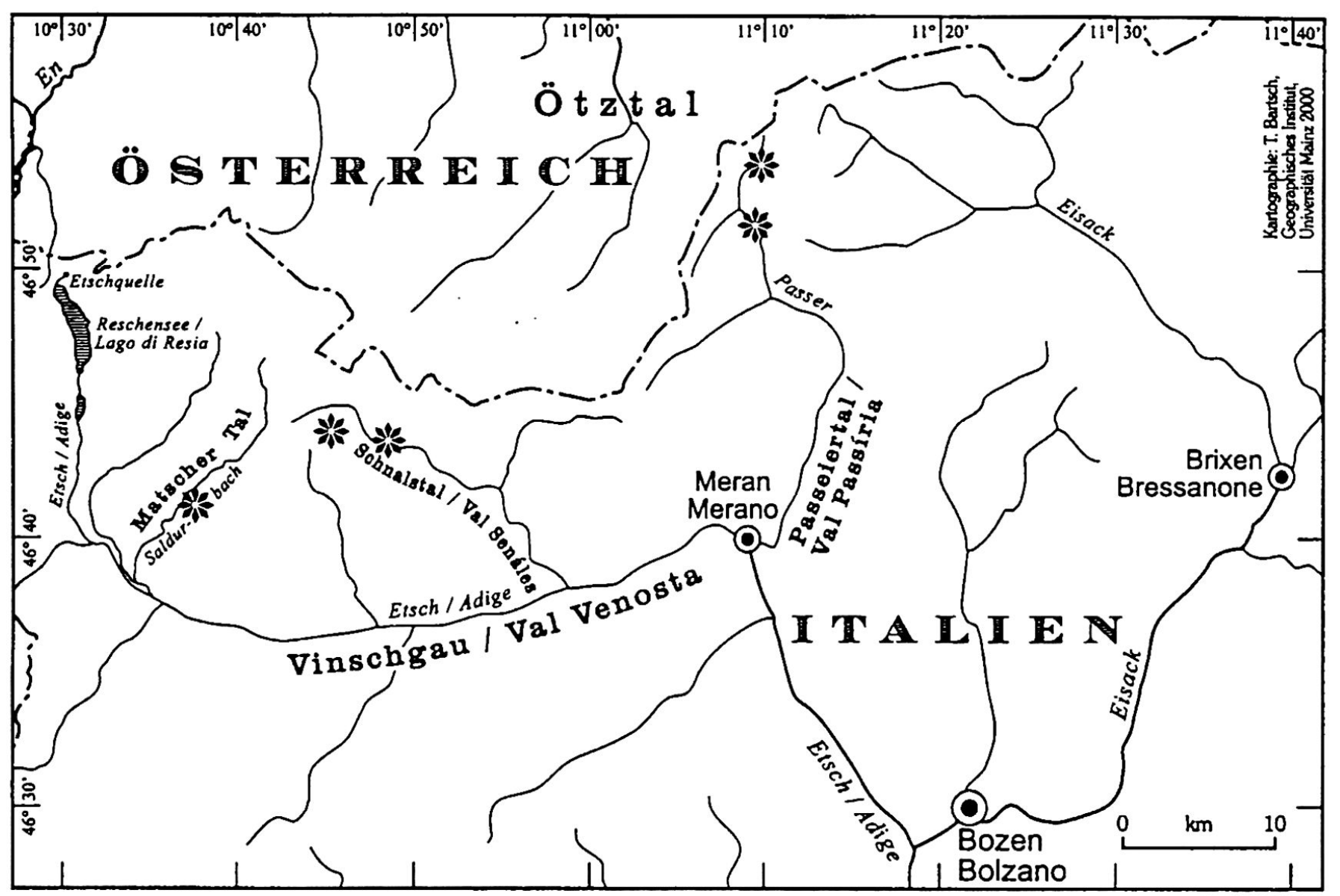

Abb. 1: Übersichtskarte der Ötztaler Alpen in Südtirol; die untersuchten Standorte sind mit Sternen bezeichnet. Map of the Ötztaler Alps in South Tyrol. Asterisks mark the sites investigated.

Aperçu cartographique des Alpes de l'Ötztal (Tirol méridional); les lieux étudiés sont marqués par une étoile. Kartographie: T. BARTSCH, Geographisches Institut, Universität Mainz

abgeschätzt. Dabei sind die Niederschläge ausgesprochen gering, so dass in den tieferen Lagen kaltgetöntes Steppenklima vorherrscht. Da Bäume noch nicht aus ihren eiszeitlichen Refugialräumen eingewandert sind, ist anspruchslose Rohbodenvegetation für die montane Höhenstufe kennzeichnend. Im Übergang zum Bølling bessert sich das Klima markant. Dadurch kommt es zum starken Anstieg der Temperatur und damit auch der Schneegrenze, die nur mehr 300 Höhenmeter unter der heutigen liegt. Die Vegetation der Ötztaler Alpen verdichtet sich zu alpinem Rasen und die untersten Höhenstufen sind mit Vorwaldgesellschaften aus beispielsweise Weide und Sanddorn bestanden. Spätestens im Aller $\varnothing$ d dominieren Kiefern- und Birkenwälder. Dabei tritt sowohl die Waldkiefer als auch die Legföhre in Erscheinung. Die bis auf $2000 \mathrm{~m}$ ansteigende Waldgrenze und die subalpine Höhenstufe wird von Zirbe und Lärche eingenommen. Während der Jüngeren Dryas ereignet sich der letzte größere Klimarückschlag mit Temperaturen um die $3 \mathrm{~K}$ unter den heutigen (BORTENSCHLAGER
1991). Für Nordtirol schätzt Kerschner (1980) die Niederschläge um $20-40 \%$ unter den heutigen. Diese klimatische Ungunstphase spiegelt sich in großen Moränenzügen im gesamten Alpenraum wider. Die Waldgrenze sinkt um 100-200 Höhenmeter gegenüber dem Allerød und Pioniergewächse treten temporär verstärkt auf.

Im Holozän sind nur geringe Temperaturschwankungen um $\pm 1 \mathrm{~K}$ zu konstatieren mit einem warmfeuchten Optimum im Atlantikum und in der Folge einem Waldgrenzhöchststand bei $2350 \mathrm{~m}$ ü.M. Die wichtigste Veränderung in der Vegetation ist die Einwanderung der sehr konkurrenzfähigen Fichte aus Südosteuropa, die in der Folge die Areale von Zirbe und Lärche auf die Waldgrenzregion einengt. Diese homogene Vegetationsentwicklung der Ötztaler Alpen wird am Nordrand - vom Inntal ausgehend - durch die Dominanz von Eichenmischwäldern differenziert. Am Südrand der Ötztaler Alpen tritt die Tanne verstärkt in Erscheinung. 


\begin{tabular}{|c|c|c|c|c|c|c|}
\hline $\begin{array}{l}\text { ka BP } \\
\text { (konv.) }\end{array}$ & Chronozonen & $\begin{array}{c}\text { Temperatur } \\
\text { (Sommerhalbjahr) }\end{array}$ & $\begin{array}{l}\text { Niederschlag } \\
\text { (Jahresmittel) }\end{array}$ & Schneegrenze & Montan (Subalpin) & Waldgrenze \\
\hline $0-2,5$ & Subatlantikum & $\leftrightarrow$ & & $\Leftrightarrow$ & Fichte (Zirbe-Lärche) & $2200 \mathrm{~m}$ \\
\hline $2,5-5$ & Subboreal & $\leftrightarrow$ & & $\Leftrightarrow$ & Fichte (Zirbe-Lärche) & $2350 \mathrm{~m}$ \\
\hline $5-8$ & Atlantikum & $\boldsymbol{T}$ & & $\Leftrightarrow$ & Fichte (Zirbe-Lärche) & $2350 \mathrm{~m}$ \\
\hline $8-9$ & Boreal & $\leftrightarrow$ & 1 & $\Leftrightarrow$ & Kiefer-Birke (Zirbe-Lärche) & $2200 \mathrm{~m}$ \\
\hline $9-10$ & Präboreal & $\downarrow$ & 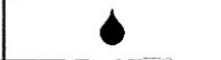 & $\sqrt{3}$ & Kiefer-Birke (Zirbe-Lärche) & $2150 \mathrm{~m}$ \\
\hline $10-11$ & Jüngere Dryas & 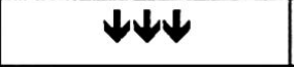 & 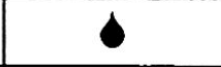 & オூス & Kiefer-Birke (Zirbe-Lärche) & $1850 \mathrm{~m}$ \\
\hline $11-12$ & Allerød & $\downarrow \downarrow$ & $?$ & $\sqrt{3}$ & Kiefer-Birke (Zirbe-Lärche) & $2000 \mathrm{~m}$ \\
\hline $12-13$ & Bølling & $\downarrow \downarrow$ & $?$ & 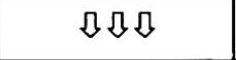 & Pionierrasen & - \\
\hline $13-15$ & Älteste Dryas & 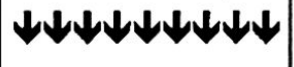 & $\bullet$ & 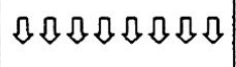 & Rohbodenvegetation & - \\
\hline
\end{tabular}

$\leftrightarrow \leftrightarrow \Leftrightarrow$ Vergleichbar mit rezenter Temperatur bzw. Schneegrenze

$\downarrow$ Temperatur $1 \mathrm{~K}$ unter rezenter Sommertemperatur

个 Temperatur $1 \mathrm{~K}$ über rezenter Sommertemperatur

- Tropfengrösse entspricht Niederschlag (qualitative Skala)

$\S$ Schneegrenze 100 Höhenmeter unter rezenter klimatischer Schneegrenze

Abb. 2: Übersicht zur Klima- und Vegetationsgeschichte der Ötztaler Alpen im Spätglazial und Holozän Development of climate and vegetation of the Ötztaler Alps during the Late-Glacial and Holocene periods Aperçu de l'évolution climatique et de la végétation dans les Alpes de l'Ötztal à la fin de l'époque glaciaire et durant l'holocène Quelle: nach Bortenschlager 1984, 1991; Nothegger 1997; Patzelt 1995; Stumböck 1999; ergänzt nach Burga \& PerRet 1998

\section{Anthropogene Umweltveränderungen ab dem Neolithikum}

In allen untersuchten Sedimenten am Südabfall der Ötztaler Alpen manifestieren sich anthropogene Eingriffe in einer Zunahme der Kulturzeigerpflanzen bei einem unter starken Schwankungen generellen Rückgang der Bewaldung (Sтимвӧск 1999). Bedingt durch die Höhenlage der untersuchten Standorte sind vor allem die Brandrodung der Wälder, die zum Teil in soliden Holzkohleschichten konserviert ist, und die Ausdehnung von Weiden- und Wiesenflächen signifikant. Waldbrand im Ostalpenraum wurde von verschiedenen Autoren bereits seit dem frühen Holozän nachgewiesen (Kofler 1992; OegGl \& WaHLMüLLER 1992). Allerdings wird auch die Schwierigkeit betont, anthropogen verursachte Brände sicher zu diagnostizieren. Jedoch spricht m.E. die starke Häufung ab dem späten Atlantikum deutlich für vorherrschenden menschlichen Einfluß.

Die frühesten Spuren des Menschen sind im inneren Schnalstal in spätneolithischen Brandrodungen nachweisbar (Hangmoor $700 \mathrm{~m}$ ESE des Marchegghofs; $10^{\circ} 48^{\prime}$ ö.L., $46^{\circ} 44^{\prime}$ n. Br.). Das diesbezügliche Radiokarbondatum weist mit $4140 \mathrm{v}$. Chr. (kalibriert und gemittelt) ein um 1000 Jahre höheres Alter als der Mann vom Hauslabjoch («Ötzi») auf, dessen Fundort sich am Alpenhauptkamm in nur $5 \mathrm{~km}$ Entfernung befindet. Die Hochlagen der Südtiroler Ötztaler Alpen wurden demnach bereits im beginnenden Spätneolithikum genutzt. Es schließen sich weitere Rodungsphasen 
an, die der Bronze-Eisenzeit und der historischen Zeit zuzuordnen sind. Im Passeiertal dagegen ist der früheste menschliche Eingriff erst zur Eisenzeit belegt. Die nächste Hauptrodungsphase erfolgt dann im frühen Hochmittelalter.

Die obgenannten Befunde lassen sich weitgehend mit dem Nordteil der Ötztaler Alpen (Patzelt et al. 1997) parallelisieren. Auch dort wurden bislang keine paläolithischen Siedlungsspuren gefunden. Bereits für das Mesolithikum können jedoch Feuerstellen bis in die alpinen Hochlagen nachgewiesen werden. Ab dem Neolithikum ist dann die Weidenutzung der natürlich waldfreien alpinen Rasen und die von dieser Höhenstufe aus erfolgte sukzessive Kulturnahme der tiefer gelegenen Wälder signifikant. Während der späten Eisenzeit und der Römerzeit werden auch die abgelegenen Seitentäler urbar gemacht. Im Spätmittelalter bedingt der zunehmende Bevölkerungsdruck aus dem Inntal und dem Vinschgau die maximale Ausdehnung des Dauersiedlungsraums und dies vor allem über Schwaighöfe, die allerdings zum großen Teil bereits in der frühen Neuzeit in saisonal bewirtschaftete Almen überführt werden.

\section{Alternierende Phasen von Kulturnahme und Bewaldung in der montanen Höhenstufe in historischer Zeit}

Standort: Als Beispiel für die montane Höhenstufe wurde ein kleinflächiges Röhricht im mittleren Matscher Tal auf $1480 \mathrm{~m}$ Höhe ausgewählt (Abb. 1). Das im Zentrum überstaute, von Schilf bestandene Röhricht «Fischweiher» geht infolge intensiver Bewirtschaftung scharf in die benachbarten Mähwiesen über, die auch die südexponierten Hänge prägen. Dagegen werden nordexponierte Hänge von Lärchenwäldern mit geringeren Anteilen an Fichte dominiert. Die Sedimente des Röhrichts sind bis zu 1,75 m tief entwickelt und ca. 2000 Jahre alt.

Methodik: Auf der Grundlage von detaillierten Pollenanalysen wurden charakteristische Pollenspektren zusammengefaßt, die alternierende Phasen von Kulturnahme und Bewaldung widerspiegeln. Ausgewählt wurden die Pollenspektren der drei höhenstufentypischen Gehölze Fichte, Lärche und (Grün-) Erle sowie wichtiger primärer und sekundärer Kulturzeiger. Die Summe aller dieser Pollentypen ergeben jeweils 100\%. Die Auswahl der Weidezeiger folgt OegGL (1994).

Die Zeitstellung der Pollenspektren wurde anhand der verfügbaren Radiokarbondaten linear interpoliert vorgenommen. Dabei ist einschränkend zu sagen, dass Radiokarbondaten keine jahrgenaue Zuordnung ermöglichen können. So ergab die jüngste datierte
Probe ein konventionelles Alter von 206 a BP bei einem Fehler von \pm 40 Jahren. Aufgrund der unperiodischen Schwankungen der ${ }^{14} \mathrm{C}$-Konzentration in der Atmosphäre wurde für diese Probe ein dendrochronologisch kalibriertes, wahres Alter von 1658-1682 und 1748-1805 n. Chr. errechnet (R. J. Van de Graaff Laboratorium der Universität Utrecht). Außerdem liegt das obgenannte konventionelle Alter nur mit der Standardabweichung von $1 \sigma(68,3 \%$ Sicherheitswahrscheinlichkeit) im Fehlerbereich von \pm 40 Jahren. Legt man $2 \sigma$ und damit $95,5 \%$ Wahrscheinlichkeit zugrunde, dann erhöht sich auch das kalibrierte Intervall beträchtlich. Daher ist die Zeitstellung der Kulturnahme- und Bewaldungsphasen nur als Orientierung zu verstehen.

Diskussion: Charakteristisch für die Pollenspektren sind die fünf prononcierten Phasen der Kulturnahme, die sich mit vier Phasen der Wiederbewaldung abwechseln. Die ältest erhaltenen Sedimente belegen deutlich Kultureinfluss bereits kurz vor der Zeitwende, also zur Römischen Kaiserzeit. In dieser Zeit wird das mittlere Matscher Tal durch eine Kulturlandschaft mit ausgedehnten Wiesen und Weiden geprägt, die sich in hohen Anteilen von Süßgräser- und Weidezeigerpollen ausdrücken. Getreideanbau ist ebenfalls signifikant nachweisbar. Vor allem der thermisch anspruchslose, relativ winterharte Roggen ist immer mit mehreren Prozent vertreten. Die anspruchsvolleren Getreidearten Weizen und Gerste dürften aber ebenfalls - wenn auch in geringerem Umfang - die Äcker bestanden haben. So ist im benachbarten Venter Tal noch im Jahr 1880 Gerste bis in eine Höhe von $1800 \mathrm{~m}$ angebaut worden (Heuberger 1975). Im Matscher Tal war wohl infolge des Klimas nur der Anbau von Sommergetreide möglich. Denn am Beispiel des äußeren Ötztals ist belegt, dass das Wintergetreide durch Früh- und Spätfröste nur an besonders begünstigten Standorten bis in eine Höhe von $1350 \mathrm{~m}$ gedeiht (Heuberger 1975).

Die typischen Baum- und Straucharten spielen in dieser Kulturlandschaft eine nur geringe Rolle. Vor allem die Fichte, die die natürliche Schlusswaldgesellschaft der montanen Höhenstufe absolut dominiert, wurde offensichtlich bereits vor der Zeitenwende im Matscher Tal in extremen Ausmaßen gerodet. Die hohen Anteile von Holzkohlepartikeln in den Sedimenten sprechen dabei für Brandrodung. Die Pollencharakteristik von Fichtenwäldern, die noch nicht unter menschlichem Einfluss standen, ist für das mittlere Atlantikum (ca. 5500 v. Chr.) exemplifiziert. Die Daten stammen aus einem Niedermoor der subalpinen Höhenstufe des Passeiertals.

Um das Jahr Null fallen größere Teile des mittleren Matscher Tals wüst. Es kommt zum starken Abfall, 
zum Teil auch zum Aussetzen der Kulturzeiger und einem Anstieg der Gehölzpollen. Dies betrifft vor allem die lichtbedürftige Lärche, eine in der Jugend schnellwüchsige und stark fruktifizierende Baumart, die sich auf Kahlflächen rasch einstellt. Die Grünerle tritt als Pionierstrauch der waldfreien Gebiete und an Lawinenhängen in Erscheinung. Die Fichte zeigt in dieser Phase keinen signifikanten Anstieg. Unmittelbar anschließend nimmt der Anteil der Fichtenpollen auf etwa das Doppelte zu. Darin spiegelt sich die Unterwanderung der Lärche durch die Schattholzart Fichte wider.

Die diskutierten Phasen wiederholen sich in der Folge mehrfach mit weitgehend gleichen Pollenspektren. Während der Römischen Kaiserzeit und der Völkerwanderungszeit bis zum beginnenden Frühmittelalter (bis ca. $600 \mathrm{n}$. Chr.) lassen sich drei Phasen der Kulturnahme konstatieren, die sich mit drei Waldregenerationsphasen abwechseln. Die drastisch gegensätzlichen Pollenspektren sprechen für eine jeweils großfächige und tiefgreifende Veränderung in der Vegetation. Daher ist für diese Zeit nicht von einer stabilen Besiedlung des Matscher Tals auszugehen. In Ansätzen Vergleichbares wurde für das Schweizer Unterengadin von ZoLLER et al. (1996) festgestellt. Dort tritt ab etwa $300 \mathrm{n}$. Chr. eine Zeit hoher Bewaldungsdichte auf, die summarisch als frühmittelalterliche Stagnation bezeichnet wird. Die dort genannten vegetationsgeschichtlichen Kriterien lassen sich auch im Pollendiagramm des Fischweihers nachweisen, wenn auch nicht in derselben Kontinuität.

Im Frühmittelalter wird der Wald noch einmal großflächig gerodet. Das gesamte Mittelalter ist dann von einem relativ kontinuierlichen Pollenspektrum gekennzeichnet, das eine dauerhafte Besiedlung nahelegt. Somit findet die für Deutschland so charakteristische spätmittelalterliche Agrardepression mit ihren umfangreichen Wüstungen (ABEL 1978) im Matscher Tal keine Entsprechung. Auch WelteN (1982) konstatiert für das Unterengadin eine großflächige Waldvernichtung und die Umgestaltung der Landschaft vor allem im Mittelalter. Zu dieser Zeit mehren sich die Zeichen von Weidgang, Getreideanbau und Brand.

In der Neuzeit (17./18. Jahrhundert n. Chr.) findet eine letzte, allerdings nicht sehr ausgeprägte Wiederbewaldung statt. Diese Wüstungsperiode ist archivalisch nicht dezidiert nachweisbar. Allerdings war das Matscher Tal im fraglichen Zeitraum Schauplatz größerer Katastrophen. 1635 wütete dort die Pest und 1781 ereignete sich im unweit gelegenen Ort Matsch ein verheerender Brand (RAMPOLD 1991), der sich wohl im starken Aufkommen der Holzkohle in den Sedimenten niedergeschlagen hat. Als Folge dieser neuzeitlichen
Katastrophen könnten zeitweise auch größere Areale brachgefallen sein. Eine andere Erklärung bietet die sogenannte «Kleine Eiszeit». Nach FlohN (1985) verbindet sich damit ein Zeitraum ungünstiger Klimabedingungen zwischen 1550 und $1850 \mathrm{n}$. Chr., der sich vereinfacht in Gletschervorstößen, einem Rückgang der Wintertemperaturen und kaltnassen Sommern ausgedrückt hat. So wird berichtet, dass die Bewohner des benachbarten Schnalstals im Jahre 1601 «viel härter als früher wirtschaften müßten, da ... durch die Zunahme der Ferner Vieh, Güter und Weiden verwildert und verdorben würden» (RICHTER 1892 aus LoosE 1984: 45). Nach Loose (1984) ist für die Kleine Eiszeit belegt, dass in verschiedenen Hochtälern Südtirols das Getreide nicht mehr ausreifte. Die daraus resultierenden Missernten konnten allerdings nicht durch einen Zuwachs an Vieh kompensiert werden, da Almen und Wiesen ebenfalls einen stark verminderten Ertrag erbrachten. Die Entsiedlung der Hochlagen in der Neuzeit steht sicher in ursächlichem Zusammenhang mit dem ungünstigen Klima. Allerdings hat dieses Klima auch zu einer unzureichenden Ernährungslage und damit zu einer Schwächung der Konstitution großer Bevölkerungsteile beigetragen und dadurch indirekt der obgenannten Pest und anderen Epidemien den Weg geebnet.

\section{Auswirkungen von Rodung und Erzabbau auf die Bewaldung der subalpinen Höhenstufe im Mittel- alter und in der frühen Neuzeit}

Erzabbau im Passeiertal: Das innere Passeiertal war mindestens seit dem beginnenden Spätmittelalter durch Erzabbau gekennzeichnet. Das $2350 \mathrm{~m}$ hoch gelegene, bedeutende Bergwerk am Schneeberg wurde 1237 n. Chr. im Bozener Notarbuch erstmals erwähnt und erst 1986, also 750 Jahre später, aufgelassen (Voelckel 1989). Zunächst wurde vor allem Silber, später auch Blei und Zink gefördert. Zum Abbau des Erzes wurde Gruben- und Brennholz benötigt. Die Verhüttung, die nach TASSER (1994) in den tieferen Lagen des Passeiertals sowie des benachbarten Ridnauntals durchgeführt wurde, erforderte zudem viel Röstholz und Holzkohle. All dies bedeutete einen hohen Holzbedarf aus der näheren Umgebung und damit einschneidende Veränderungen in der Vegetation.

Standort: $3 \mathrm{~km}$ vom Bergwerk entfernt befindet sich das Niedermoor «Kurzmoos» auf $1820 \mathrm{~m} \mathrm{NN}$ (Abb. 1). Die subalpinen Wälder der Umgebung bestehen aus Lärchen und Fichten, die im Unterwuchs vor allem von Gräsern und Heidekrautgewächsen begleitet werden. Die $5 \mathrm{~m}$ tiefen Sedimente des Kurzmooses wurden beprobt und paläoökologisch untersucht (s. Sтимвӧск 1999). 
Diskussion: Es zeigt sich, dass bereits um die Zeitenwende die höhenstufentypischen dichten Fichtenwälder zum größeren Teil gerodet wurden. Die freien Flächen werden beweidet oder verbuschen mit der anspruchslosen Grünerle, die nach Zoller et al. (1996) breite Verwendung für Fütterung, Einstreu und Feuerholz fand. Ab etwa 400 n. Chr. tritt die auch im schweizerischen Unterengadin konstatierte frühmittelalterliche Stagnation (ZOLLER et al. 1996) mit regenerierten Fichtenwäldern in Erscheinung, die bis etwa 1000 n. Chr. andauert. Für das gesamte Frühmittelalter einschließlich dem beginnenden Hochmittelalter ist also von lediglich minimalen menschlichen Einflüssen in der subalpinen Höhenstufe des Passeiertals auszugehen.

Ab 1000 n. Chr. kommt es zu einer erneuten Hauptrodungsphase der Fichtenwälder, wobei die hohen Holzkohleanteile in den Sedimenten deutlich für Brandrodung sprechen. Somit wird das Hoch- und Spätmittelalter durch eine Kulturlandschaft mit einem Mosaik aus Weiden, Wiesen und Fichtenwäldern geprägt unter Beteiligung der anthropogen geförderten Pionierholzart Lärche. Unterstützung findet diese Interpretation durch Heuberger (1975), der im selben Zeitraum für das Ötztal eine starke horizontale und vertikale Ausdehnung des Dauersiedlungsraums konstatiert. In diese Zeitphase fällt auch der Anstieg der Getreidepollen. Allerdings ist nicht zu erwarten, dass in näherer Umgebung um das Kurzmoos Getreide in nennenswertem Umfang angebaut wurde. Dagegen sprechen die nur geringen Prozentwerte in Verbindung mit der guten Fernflugfähigkeit der Getreidepollen. So sind in den Zentralalpen bis in hochalpine Lagen Getreidepollen mit über 1\% Anteil dokumentiert worden (Burga \& Perret 1998; Stumböck 1999). Im inneren Passeiertal liegt die rezente maximale Höhe des Getreideanbaus zwischen 1600 und $1700 \mathrm{~m}$ ü.M. (FISCHER 1974). Selbst wenn zur Zeit des mittelalterlichen Landesausbaus die Anbaugrenze etwas höher gelegen haben mag, so können sich diese kleinen Flächen kaum im Pollendiagramm niedergeschlagen haben.

Die hochmittelalterliche Rodung hat offensichtlich erheblich in das ökosystemare Gefüge des Kurzmooses eingegriffen. Seit dem mittleren Atlantikum 7000 Jahre lang - wurde der Standort selbst von Fichtenwäldern dominiert. Entsprechende Waldtorfe und Holzfunde belegen dies. Mit den menschlichen Eingriffen kommt es ab 1000 n. Chr. zur starken Vernässung und Ansiedlung von Sauergräsern. Kahlschlag, insbesondere von Nadelbäumen, hat erhebliche Auswirkungen auf den Wasserhaushalt durch die drastische Verminderung von Interzeption und Transpiration pro Flächeneinheit (BURSCHEL \& Huss 1987). Dies führt zu hohem oberflächlichem Abfluß an den
Hängen und zu einem erheblichen Wasserüberschuss in den Senken. Das so entstandene Niedermoor ist daher nicht als natürlich, sondern als anthropogene Folgevegetation zu werten.

Ob die sich ab 1000 n. Chr. abzeichnenden Veränderungen in der Vegetation auch bereits auf den Erzabbau am Schneeberg zurückgeführt werden müssen, lässt sich nicht entscheiden. Entsprechende Archivalien stehen nicht zur Verfügung. In der frühen Neuzeit allerdings legt die Archivlage einen solchen Schluss nahe. Aus dem Jahr 1545 n. Chr. ist eine Waldordnung bekannt, die Wälder, aus denen Holz für das Bergwerk geschlägert werden darf, ausweist (TASSER 1994). Dazu zählen auch die Gebiete um das Kurzmoos. Das Jahr $1545 \mathrm{n}$. Chr. entspricht in der Untersuchung etwa dem gemittelten kalibrierten Radiokarbondatum 366 v.h. (1519-1574 und 1626-1648 n. Chr.). Dieser Abschnitt ist gekennzeichnet durch wiederholte Einbrüche der Fichte, die kombiniert sind mit Holzkohlemaxima, die beispielsweise aus der Köhlerei oder aus dem Abbrennen geschlägerter Flächen stammen können. Zudem sind zwei Grobsandbänder eingeschwemmt einzigartig in der gesamten Sedimentsäule des Kurzmooses, die sich kontinuierlich seit dem Spätglazial entwickelt hat. Alle diese Befunde sprechen für erhebliche Waldverwüstung im inneren Passeiertal im 16. Jahrhundert infolge des Erzabbaus - begleitet von Erosionserscheinungen an den gerodeten Hängen. Der Nutzungsdruck auf die subalpinen und montanen Wälder muss in dieser Zeit erheblich gewesen sein, da die zweite Hälfte des 15. und die erste Hälfte des 16. Jahrhunderts als die höchste Blütezeit des Bergbaus am Schneeberg mit bis zu 1000 Knappen überliefert ist.

\section{Resümee}

Die in Kap. 6 und 7 diskutierten anthropogenen Umweltveränderungen sind lediglich Fallbeispiele aus den Südtiroler Ötztaler Alpen. Wünschenswert wäre die Übertragung dieser Befunde auf einen größeren Raum. Einer solchen Regionalisierung steht allerdings ein erhebliches Forschungsdefizit entgegen. Bis in die achtziger Jahre standen Fragen der Klimaentwicklung, der Waldgrenze und der postglazialen Einwanderung der Gehölze im Vordergrund. Erst dann hat sich der Schwerpunkt der Forschung auf den menschlichen Einfluss verlagert - nicht zuletzt unter dem Eindruck des Fundes des Gletschermannes vom Hauslabjoch im September 1991. Zudem ist das Wirken des Menschen im Gegensatz zu den natürlichen Umweltveränderungen weder zeitlich noch räumlich kontinuierlich, sondern höchst selektiv und phasenhaft. Daher lassen sich die bislang vorliegenden Ergebnisse nicht verallgemeinern. 
Zur Zeit werden weitere Projekte durchgeführt, z.B. die «Ötztalstudie» (Patzelt 1996; Patzelt et al. 1997) oder Untersuchungen im inneren Passeiertal (STUмBöcK 1999). Diese werden sicher die Datenbasis entsprechend ausweiten. Damit ist zu erwarten, dass zu einem späteren Zeitpunkt die Ergebnisse auch über den Ötztaler Raum hinaus übertragen werden können.

\section{Dank}

Der Verfasser dankt der Deutschen Forschungsgemeinschaft (DFG) für die Finanzierung dieser Arbeit.

\section{Literatur}

ABEL, W. (1978): Geschichte der deutschen Landwirtschaft. - 3. Auflage, Stuttgart: Eugen Ulmer.

AMT FÜR ÜBERÖRTLICHE RAUMORDNUNG: Orthofotokarte der Autonomen Provinz Bozen-Südtirol 1:10.000, Nr. 606, 607, 610, 611, 614, 615, 1112, 1202, 1205, 1206, 1210, 1211, 1216, 1301, 1303, 1305, 1306.

Biologisches Landeslabor (Hrsg.) (1991): Kataster der Moore und Feuchtgebiete Südtirols. - = Tätigkeitsbericht Biologisches Landeslabor Autonome Provinz Bozen 6: 1-214.

BortensCHLager, S. (1984): Beiträge zur Vegetationsgeschichte Tirols I. Inneres Ötztal und unteres Inntal. - In: Berichte naturwissenschaftlich-medizinischer Verein Innsbruck 71:19-56.

Bortenschlager, S. (1991): Die Waldgrenze im Postglazial. - In: Kovar-Eder, J. (Hrsg.): Palaeovegetational development in Europe and regions relevant to its palaeofloristic evolution. - Wien: 9-13.

Burga, C.A. \& R. Perret (1998): Vegetation und Klima der Schweiz seit dem jüngeren Eiszeitalter. - Thun: Ott.

Burschel, P. \& J. Huss (1987): Grundriß des Waldbaus. Ein Leitfaden für Studium und Praxis. - Hamburg, Berlin: Paul Parey.

Fischer, K. (1974): Das Passeiertal. Grundzüge einer geographischen Landeskunde. - In: Der Schlern 48: 344-380.

FLOHN, H. (1985): Das Problem der Klimaänderungen in Vergangenheit und Zukunft. - Darmstadt: Wissenschaftliche Buchgesellschaft.

HantKe, R. (1983): Eiszeitalter. Die jüngste Erdgeschichte der Schweiz und ihrer Nachbargebiete, Teil 3.

- Thun: Ott.

Heuberger, H. (1975): Das Ötztal. - In: Innsbrucker Geographische Studien 2: 213-249.

Hydrographisches Amt (1994): Precipitazioni 70 anni di osservazioni in Alto Adige 1921-1990-Niederschläge 70 Jahre Beobachtungen in Südtirol 1921-1990. - Autonome Provinz Bozen-Südtirol: Eigenverlag.

Hydrographisches Amt (1995): Annale idrologico Hydrologisches Jahrbuch 1991. - Autonome Provinz Bozen-Südtirol: Eigenverlag.
Kerschner, H. (1980): Outlines of the climate during the Egesen advance (Younger Dryas, 11000-10000 BP) in the Central Alps of the Western Tyrol, Austria. In: Zeitschrift für Gletscherkunde und Glazialgeologie 16: 229-240.

Kofler, W. (1992): Die Vegetationsentwicklung im Spätpaläolithikum und Mesolithikum im Raume Trient. - In: Museo Tridentino di Scienze Naturali - Preistoria Alpina 28: 83-103.

Loose, R. (1984): Die «Kleine Eiszeit» und die Folgen für Siedlung und Wirtschaft in den Alpen. - In: Mannheimer Berichte aus Forschung und Lehre 24: 40-48.

Lunz, R. (1986): Vor- und Frühgeschichte Südtirols. Band 1 Steinzeit. - Bruneck: Eigenverlag.

Merkt, J., Lüttig, G. \& H. Schneekloth (1971): Vorschlag zur Gliederung und Definition der limnischen Sedimente. - In: Geologisches Jahrbuch 89: 607-623.

Moore, P.D., WebB, J.A. \& M.E. Collinson (1991): Pollen Analysis. - 2. Auflage, Oxford: Blackwell.

Nothegger, B. (1997): Palynologische Untersuchungen zur Ermittlung von Waldgrenz- und Klimaschwankungen in den Ostalpen anhand der Profile Schönwies und Rotmoos. - Unveröffentlichte Diplomarbeit am Botanischen Institut der Universität Innsbruck.

OEgGL, K. (1994): The palynological record of human impact on highland zone ecosystems. - In: BIAGI, P. \& J. NANDRIS (Hrsg.): Highland zone exploitation in southern Europe. - In: Monografie di «Natura Bresciana» 20: 107-122.

OegGl, K. \& N. Wahlmüller (1992): Vegetation and climate history of a high alpine mesolithic camp site in the Eastern Alps. - In: Museo Tridentino di Scienze Naturali - Preistoria Alpina 28: 71-82.

Ozenda, P. (1988): Die Vegetation der Alpen im europäischen Gebirgsraum. - Stuttgart, New York: Gustav Fischer.

Patzelt, G. (1975): Unterinntal - Zillertal - Pinzgau - Kitzbühel. Spät- und postglaziale Landschaftsentwicklung. - In: Innsbrucker Geographische Studien 2: 309-329.

Patzelt, G. (1995): Holocene glacier and climate variations. - In: SCHIRMER, W. (Hrsg.): Quaternary field trips in Central Europe Vol. I. - München: Friedrich Pfeil.

Patzelt, G. (1996): Modellstudie Ötztal - Landschaftsgeschichte im Hochgebirgsraum. - In: Mitteilungen Österreichische Gesellschaft, Wien 138: 53-70.

Patzelt, G., Kofler, W. \& B. Wahlmüller (1997): Die Ötztalstudie - Entwicklung der Landnutzung. - In: Oeggl, K., Patzelt, G. \& D. Schäfer: Alpine Vorzeit in Tirol. Begleitheft zur Ausstellung. - Innsbruck: 46-62.

RAmpold, J. (1991): Vinschgau. - = Südtiroler Landeskunde in Einzelbänden 1. - 6. Auflage, Bozen: Athesia: $1-448$.

SCHNEEKLOTH, H. \& S. SCHNeider (1972): Vorschlag zur Klassifizierung der Torfe und Moore in der Bundesrepublik Deutschland. - In: Telma 2: 57-63.

SCHWEINGRUBER, F.H.(1990): Mikroskopische Holzana- 
tomie - Anatomie Microscopique du Bois - Microscopic Wood Anatomy. - 3. Auflage, Eidgenössische Forschungsanstalt, Birmensdorf.

SтUмвӧск, M. (1999): Die spät- und postglaziale Vegetationsgeschichte des nordwestlichen Südtirols mit einem Beitrag zur Verknüpfung natürlicher Archive mit historischen Quellen. - Berlin, Stuttgart: Gebrüder Borntraeger.

TASSER, R. (1994): Das Bergwerk am Südtiroler Schneeberg. - Bozen: Athesia.

VoelcKel, H.M. (1989): Schneeberg. 800 Jahre Bergbau zwischen Ridnaun und Passeier. - Ratschings, Moos in Passeier: Eigenverlag.

Welten, M. (1982): Pollenanalytische Untersuchungen zur Vegetationsgeschichte des Schweizerischen Nationalparks. $-=$ Ergebnisse der wissenschaftlichen Untersuchungen im Schweizerischen Nationalpark 80, Chur: $1-43$.

Zoller, H., Erny-Rodmann, C. \& P. Punchakunnel (1996): The history of vegetation and land use in the Lower Engadine (Switzerland). Pollen record of the last 13000 years. $-=$ Nationalpark-Forschung in der Schweiz 86, Zernez: 1-65.

\section{Zusammenfassung: Anthropogene Umweltverände- rungen in den Südtiroler Ötztaler Alpen in histori- scher Zeit}

Methodische Grundlage dieser Studie sind paläoökologische Untersuchungen von Sedimenten aus Feuchtgebieten der Ötztaler Alpen in Südtirol. Schwerpunkt ist dabei das Wirken des Menschen in den letzten 2000 Jahren, das sich in der Rodung der höhenstufentypischen Wälder und in der Anlage von Kulturland widerspiegelt. Ein Beispiel aus der montanen Höhenstufe belegt die sich abwechselnden Phasen von Kulturnahme und Wiederbewaldung, die in der Römischen Kaiserzeit und der Völkerwanderungszeit eine wenig stabile Besiedlung wiedergeben. Das Mittelalter und die Neuzeit sind dagegen dauerhaft besiedelt. In einem weiteren Beispiel werden die Veränderungen der subalpinen Wälder in historischer Zeit unter besonderer Berücksichtigung des Bergbaus diskutiert. Dabei lassen sich in der frühen Neuzeit Brandrodung und Erosion mit dem intensiven Erzabbau parallelisieren.

\section{Summary: Man-made Environmental Change in the Ötztaler Alps (South Tyrol) Over the Centuries}

This study is based on palaeoecological investigations of sediments gained from fens in the Ötztaler Alps (South Tyrol). The focus is on the impact of man during the last 2000 years, as reflected by the clearing of mountain forests and establishment of cultivated areas. The alternating periods of agriculture and refor- estation found in the montane altitudes points to instable settlement phases during the first centuries AD. Since the Middle Ages, permanent settlements have been established. Further, changes in subalpine forests over the centuries are discussed with particular attention being paid to mining. Thus, the slash-and-burn methods and erosion of the early Modern Age appear to be strongly connected to intensive ore mining activities.

\section{Résumé: Modifications environnementales anthropo- gènes dans les Alpes de l'Ötztal, en Tirol méridional, à l'époque historique}

Des recherches paléoécologiques sur les sédiments dans des territoires humides des Alpes de l'Ötztal (Tirol méridional) constituent la base méthodologique de la présente étude. Celle-ci est focalisée sur l'activité humaine durant les deux derniers millénaires, une activité qui se répercute dans le défrichement en altitude des forêts et la formation de paysages humanisés. A l'époque impériale romaine et durant la période de migration des peuples, la forêt étagée donne lieu à une alternance de phases de défrichement et de phases de reforestation; cette époque historique se caractérise donc par un peuplement peu stable. Au Moyen-Âge et à l'époque moderne par contre, le peuplement est permanent. Un autre exemple montre les transformations des forêts subalpines à l'époque historique sous l'angle de l'exploitation minière. En ce qui concerne le début de l'époque moderne, il est possible de mettre en parallèle le défrichement par le feu et l'érosion d'une part, l'extraction minière intensive d'autre part.

PD Dr. Max Stumböck, Geographisches Institut der Universität Augsburg, Universitätsstrasse 10, D-86135 Augsburg.

e-mail:Max.Stumboeck@t-online.de

Manuskripteingang/received/manuscrit entré le 5.5.2002

Annahme zum Druck/accepted for publication/accepté pour l'impression: 20.9.2002 